\title{
Erratum to: Slime Mould Memristors
}

\author{
Ella Gale • Andrew Adamatzky • Ben de Lacy Costello
}

Published online: 11 December 2014

(C) Springer Science+Business Media New York 2014

\section{Erratum to: BioNanoSci. \\ DOI 10.1007/s12668-014-0156-3}

The original version of this article unfortunately contained mistakes in the second and third paragraphs under Section 4 and Figure 6 legend. The changes are underlined as follows:

\section{Second and third paragraphs under Section 4:}

Figure 6 shows a circuit which could be used to model the situation: this circuit contains a one-port 'black box' which we measure. We assume that this one-port contains a voltage source, which we are symbolising as a battery because Physarum is alive and uses chemical energy to produce reactions and the motion of the membrane, and a memristor (or a memristor-resistor in series). In fact, from long-term experiments, we have seen a slow oscillation with a half-period of around $700 \mathrm{~s} \mathrm{[15]} \mathrm{that} \mathrm{could} \mathrm{fit} \mathrm{the} \mathrm{description} \mathrm{of} \mathrm{such} \mathrm{a}$ voltage source, especially as it was observed to give a current $\underline{\text { of } \approx 2 \times 10^{-9} \mathrm{~A}}$, so it is on the same order as our lower current $\bar{V}-I$ curve measurements. With the addition of an internal voltage source, we are now modelling the Physarum as an

active memristor [19] (standard memristors are passive components).

The current, $i_{q}$ is the background current from the living Physarum. From the circuit in Fig. 6, we can write the following expression for the measured current, $I_{\text {tot: }}$ :

$I_{\text {tot }}=i_{d} \pm i_{q}$

where $i_{d}$ is the current that is driven by the external voltage, $V$. The background current source can either add to or oppose the external power source, and thus, the background current is either in the same or opposite direction to the driven current, as we use + and - to represent this, where it is understood that the internal ions may not have the same charge as the electrons and we take $+i_{q}$ to be the direction of increase in total current. $V_{q}$ is the voltage associated with the internal 'battery'.

\section{Figure 6 legend:}

Fig. 6 The equivalent circuit for the Physarum memristor, the shaded area represents the middle active memristor one-port

The online version of the original article can be found at http://dx.doi.org/ 10.1007/s12668-014-0156-3.

E. Gale $(\triangle) \cdot$ A. Adamatzky $\cdot$ B. de Lacy Costello

University of the West of England, Bristol, BS16 1QY, UK

e-mail: ella.gale@gmail.com

A. Adamatzky

e-mail: andrew.adamatzky@uwe.ac.uk

Present Address:

E. Gale

Applied Maths and Science, Khalifa University of Science,

Technology and Research, P.O. Box 127788, Abu Dhabi,

United Arab Emirates 